\title{
A Fully-Programmable Neural Interface for Multi-Polar, Multi-Channel Stimulation Strategies
}

\author{
Anthony Guilvard*, Amir Eftekhar*†, Song Luan*†, Christofer Toumazou*† and Timothy G. Constandinou* ${ }^{* \dagger}$ \\ ${ }^{*}$ Department of Electrical and Electronic Engineering, Imperial College London, SW7 2BT, UK \\ ${ }^{\dagger}$ Centre for Bio-Inspired Technology, Institute of Biomedical Engineering, Imperial College London, SW7 2AZ, UK \\ Email: \{anthony.guilvard10, a.eftekhar, song.luan09, c.toumazou, t.constandinou\}@imperial.ac.uk
}

\begin{abstract}
This paper describes a novel integrated electrode interface for multi-polar stimulation of multi-electrode arrays. This interface allows for simultaneous stimulation using multiple electrodes configured as source or sink with different phase and amplitudes in order to perform field shaping inside the tissue. The system is designed in an high voltage $0.18 \mu \mathrm{m}$ CMOS process with 8 channels. It features an output voltage swing of $16 \mathrm{~V}$ and current up to $0.5 \mathrm{~mA}$ for electrode impedences of up to $30 \mathrm{k} \Omega$ which is suitable for cuff and cortical grid arrays. This electrode interface comprise a digital module which stores stimulation settings and operates the different electrode channels. Here we present the full system architecture and simulation results.
\end{abstract}

\section{INTRODUCTION}

Neural prostheses is a growing field of study for the treatment of neurological disorders. Medical implants utilising these prostheses have been successfully developed to alleviate the symptoms of Parkinson's disease, epilepsy, clinical depression and many others. These devices utilise electrical stimulation to modulate and/or suppress neuronal signalling by interfacing electronic devices to both the central and peripheral nervous system. For most of these devices, the stimulation settings are the results of clinical trials and specific adjustments for each patient [1]. So although effective, the mechanisms involved during electrical stimulation of neural tissue are not clearly understood.

Using multi-electrode arrays can provide better control of the stimulation. In particular, multi-polar configurations, in which the stimulation current is steered between several electrodes can shape the field in the tissue [2] and hence have better spatial selectivity. This type of selectivity is a key challenge in emerging medical devices, which allow unidirectional, fibre and fascicle-type selectivity and avoid unintentional excitation [3].

In addition, as the number of electrodes reaches the thousands, the ability to elicit more control and maintain device size and power consumption within acceptable safety limits for chronic use is essential. In the long term, factors such as electrode stability/degredation [4], neural fatigue and stimulation efficiency greatly affect the optimal performance of the neural prosthetic [5]. Hence, developing novel stimulation strategies is crucial both to improve prosthetic technology and to allow for implementation of stimulation strategies in medical devices.

There have been attempts to integrate efficient stimulation systems for multi-electrode arrays including [6], [7] and ref-

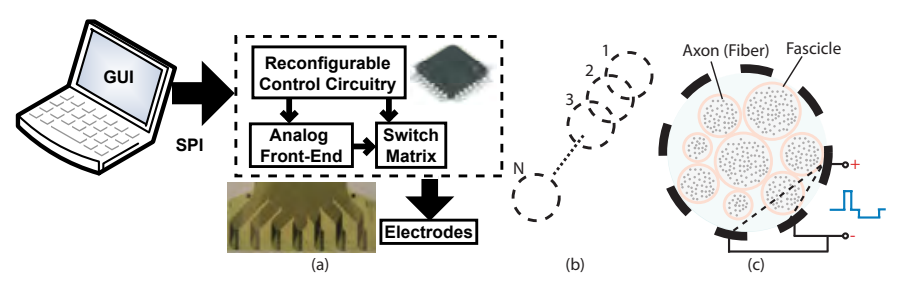

Fig. 1. System concept of (a) general architecture, (b) an example cuff multi-electrode array with multiple rings and (c) an example of programmable source/sink for field shaping electrical stimulation.

erence within. However, none have combined the programmability for different waveforms with versatile strategy for multipolar stimulation strategies. When low power consumption and very large number of channels need to be achieved, designers usually adopt stimulation strategy involving only one active electrode at the time [8], [9]. Multi-polar stimulation interfaces have been designed on-chip [10] and on PCB [11]. While PCB solutions offer very large control on the stimulation, integrated designs feature much lower size with better matching and power consumption.

In this paper we present such a device, capable of multipolar configurations and implementation of completely arbitrary stimulus waveforms. The paper is organized as followed: Section II presents the whole system, section III describes the main analogue circuits and the digital module, section IV presents simulation results of the full system and section $\mathrm{V}$ concludes the paper.

\section{SySTEM OVERVIEW}

The architecture of the system consists of a PC-based GUI for defining stimulus parameters and hardware for generating stimulus waveforms (Fig. 1). This work focuses on the main hardware implementation that allows for active control of the electrodes and arbitrary waveform generation. Fig. 1b also shows an example multi-ring multi-electrode cuff where the rings can be used as multiple sources or sinks for field shaping and selective spatial stimulation (Fig. 1c).

The system developed (Fig. 2) contains circuitry to drive a single electrode and a main controller for global stimulus control. The individual channel circuitry, contains (1) Analogue circuits to produce voltage or current stimulus (up to $500 \mu \mathrm{A}$ for electrode impedances up to $30 \mathrm{k} \Omega$ ), remain floating 


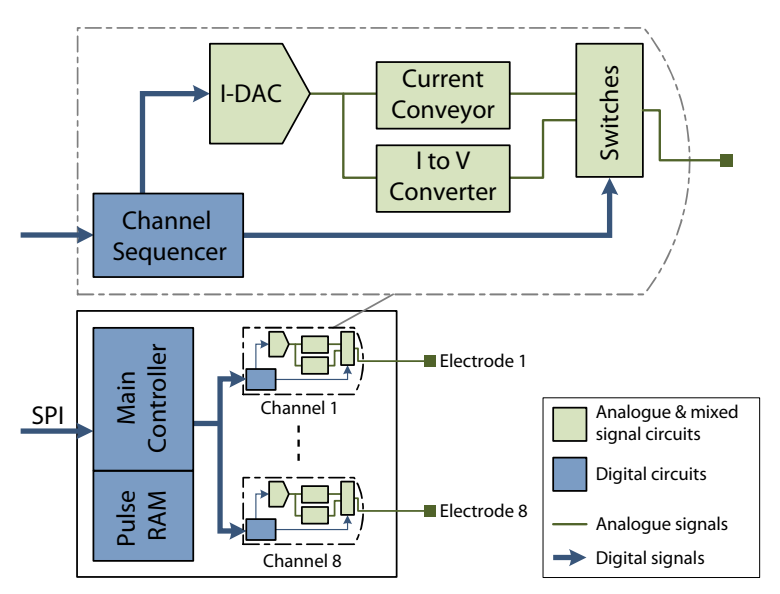

Fig. 2. Block description of the system containing the individual channel architecture (top) and the whole 8 channel system with digital control circuitry.

or act as a voltage reference and (2) Digital control circuitry, channel sequencer, for defining voltage or current stimulation, stimulus amplitude range and stimulus timings. The spatial and temporal resolution specifications were defined to be used with multi-electrode cuff electrodes [12].

The digital main controller and RAM use an SPI interface to store a custom waveform shape (which is common to all electrodes) and the individual channel specifications, which will be described in more detail later.

\section{IMPLEMENTATION}

In this section we will outline the main blocks implemented for the realisation of the analogue and digital circuitry with some of the applicable simulated results of each.

\section{A. Front end circuit}

The front end circuit is composed of a current DAC, a current conveyor, a transimpedance (current to voltage) and switches (Fig. 2). The DAC delivers a current in the range of $-100 \mu \mathrm{A}$ to $100 \mu \mathrm{A}$ which is a sub-range of the final output. This current is mirrored and amplified through a current conveyor which increases the voltage compliance to $20 \mathrm{~V}$. The DAC output can also be connected to a transimpedance in order to provide a voltage controlled output. The switches at the electrode end of the circuit are operated by the digital circuitry and define the electrode mode. The current conveyor and the transimpedence amplifier are supplied with $20 \mathrm{~V}$ to obtain the necessary voltage headroom when stimulating tissue whereas the DAC is supplied with $1.8 \mathrm{~V}$ in order to save power.

1) Current DAC: The DAC implemented in this system is based on a W-2W ladder. The W-2W ladder uses the same concept as R-2R ladder but with MOS transistors[13]. The main advantages of this type of DAC is that the ladder requires only two sizes of transistors and one bias. This feature ensures superior matching and limited use of die area which are particularly suitable for implementation of arrays [14].

In the proposed system, each channel can scale the pulse waveform. This scaling is achieved directly with the DAC

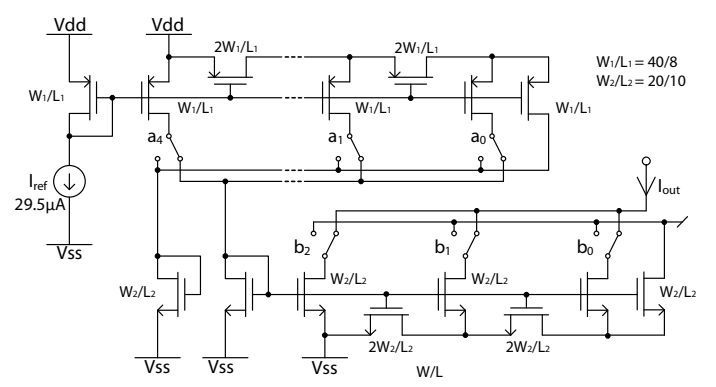

Fig. 3. Current W-2W DAC implemented using scaling through coefficients $b$ and amplitude defined by the digital input $a$.

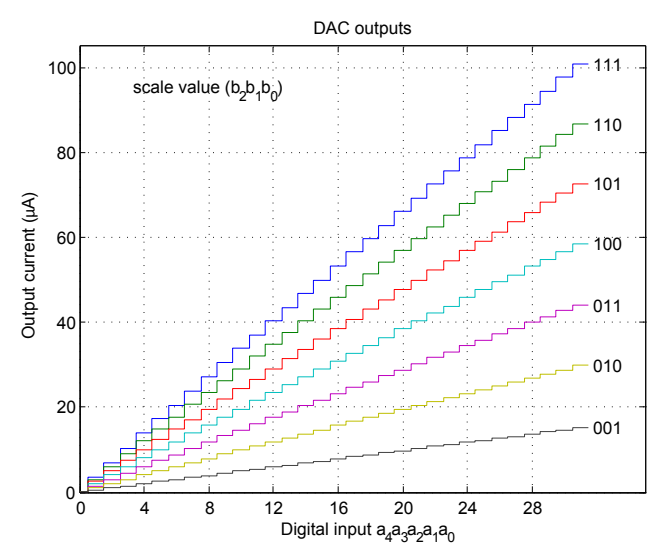

Fig. 4. DAC output when the digital input, $a_{4} a_{3} a_{2} a_{1} a_{0}$,varies and the different scales are set via $b_{2} b_{1} b_{0}$.

structure chosen (Fig. 3). This DAC consists of two W$2 \mathrm{~W}$ ladders: the p-channel ladder receives digital values of the waveform and the n-channel ladder scales the output. Consequently the final output current of the DAC is the product of two digital inputs $a_{4} a_{3} a_{2} a_{1} a_{0}$ and $b_{2} b_{1} b_{0}$ :

$$
I_{\text {out }}=I_{\text {ref }} \cdot\left(\sum_{i=0}^{4} a_{i} \cdot 2^{i-4}\right) \cdot\left(\sum_{i=0}^{2} b_{i} \cdot 2^{i-2}\right)
$$

The output of the DAC drives a current mirror which provides a sign selection: it can produce the input current as a current source or as a current sink. As mentioned the final output of the DAC can produce current from $\pm 100 \mu \mathrm{A}$ and can be scaled depending on the necessary range, as shown in Fig. 4.

2) Current conveyor: In order to apply the stimulation current produced by the DAC to the electrode at the defined current levels $(500 \mu \mathrm{A})$ and with sufficient voltage headroom $(16 \mathrm{~V})$ an output stage is required. This is achieved using a second generation current conveyor (Fig. 5). A class AB output stage is used to source both positive and negative currents. The current mirrors are cascoded to improve the output impedance. When used in the system, the voltage input (Y) is connected to ground, therefore potential of the current input $(\mathrm{X})$ is also $0 \mathrm{~V}$. The output $(\mathrm{Z})$ of the current conveyor has the opposite sign of the input $(\mathrm{X})$. 


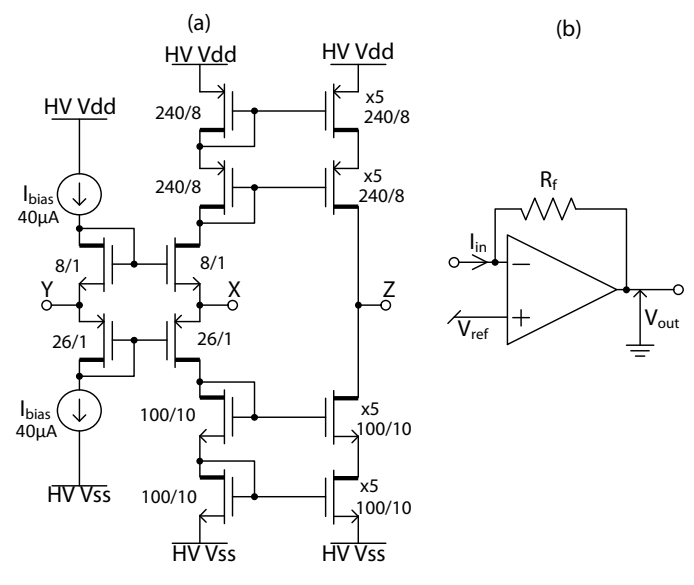

Fig. 5. The (a) current conveyor and (b) transimpedance used to generate the final current and voltage stimuli.

3) Transimpedance: In order to apply a voltage to the electrode, the output current from the DAC needs to be converted into a voltage. A two-stage operational amplifier has been implemented to perform this transimpedance function (Fig. 5). The output voltage dynamic range can be set by the feedback resistor $R_{f}(2)$.

$$
V_{\text {out }}=V_{\text {ref }}-R_{f} \cdot I_{\text {in }}
$$

where the non-inverting input, $V_{r e f}$ is connected to ground because no offset is needed in the output voltage.

\section{B. Digital controller}

The digital controller operates the analogue circuitry to produce electrical stimulations. Two entities have been designed to generate the necessary control signals: the channel sequencer and the main controller. As mentioned, the first one stores the settings related to one electrode and operates the analogue circuitry of one channel; the latter stores the pulse waveform, interpulse delay and transmits channel settings received from SPI to the individual channels.

The communication between the main controller and the channels is ensured by a single internal bus. Consequently, the main controller needs to specify the identifier of the channel before sending parameters on the bus. This communication structure simplifies the routing compared with a fully parallel communication bus between the main controller and the channels. It also eases the addition and deletion of channels as no change to the entities is required to handle less or more channels. The SPI streams structure which can be sent to the main controller are displayed in Fig. 6.

1) Principle of operation: A typical setting sequence includes the transmission of the pulse waveform and the interpulse delay followed by the loading of the settings for each channel of the system. Each operation starts with a command word and is followed by the corresponding configuration mode or to start or stop the stimulation.

When the waveform loading command is sent (wave $\mathrm{cmd}$ ), the main controller copies the following SPI stream in the

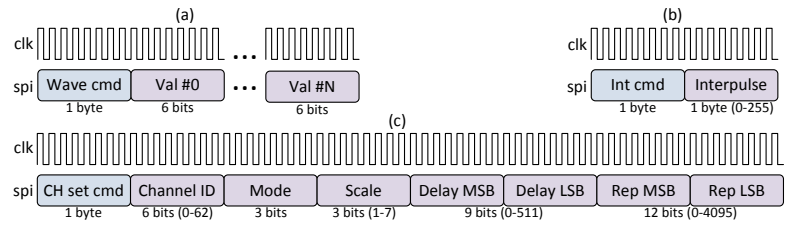

Fig. 6. SPI stream for (a) waveform loading, (b) interpulse duration loading and (c) channel settings loading.

RAM. Only the 6 LSBs of each SPI word contains waveform information. The MSB is used to notify if another waveform value will be sent in the next cycle or if the waveform has been entirely sent. When the channel settings command is sent ( $\mathrm{CH}$ set $\mathrm{cmd}$ ), the main controller first temporary stores the settings receive via SPI and then sends all these parameters via the internal bus to the channels. The channel identifier is used to activate the correct channel sequencer when settings are sent through the internal bus.

The system enters and exits stimulation mode via specific SPI commands. When an SPI start command is sent, the main controller transmits in turn a synchronous start command on the internal bus to activate all channel sequencers. Then it sends the pulse waveform on the internal bus followed by the interpulse delay and starts again until a stop command is received. When activated, channel sequencers enter the mode defined in their settings. Active channels delay incoming waveform in a buffer and apply the scale value to the DAC. They continue to output the pulse waveform until the number of repetitions is reached or until a stop command is asserted.

2) Stimulation settings: Table I lists the parameters available in the system. The pulse waveform is stored in the RAM. It consists on a list of DAC values representing the pulse shape. The interpulse delay, also stored in the RAM, is the time which separates two stimulation pulses. In this system the pulse frequency can be set from $2 \mathrm{~Hz}$ to $1 \mathrm{kHz}$.

The remaining settings are specific to every channel and are stored inside the channel sequencer. The mode of the channel defines how the channel operates during stimulation. Four active mode are available: two voltage modes (V) and two current modes (I). In order to perform multi-polar stimulation, the sign of the pulse waveform can be inverted $( \pm)$. Three passive modes are available (Vdd, Vss and gnd). These modes can be used for unipolar stimulation or to shield some areas of the tissue during stimulation. The last electrode mode is

TABLE I

STIMULATION SETTINGS STORED IN DIGITAL MODULE

\begin{tabular}{|c|c|c|}
\hline Setting (location) & Resolution & Range \\
\hline Pulse waveform (MC) & $1,023 \times 6$-bit & 0 to $1,023 \mu \mathrm{s} \times \pm 500 \mu \mathrm{A} / \pm 8 \mathrm{~V}$ \\
\hline Interpulse delay (MC) & 8-bit & 1.023 to $522 \mathrm{~ms}(2.048 \mathrm{~ms}$ steps $)$ \\
\hline Mode (CS) & 3-bit & $\pm \mathrm{V}, \pm \mathrm{I}, \mathrm{HighZ}, \mathrm{Vdd} / \mathrm{Vss} / \mathrm{Gnd}$ \\
\hline Scale (CS) & 3-bit & $1 / 7$ to $7 / 7$ \\
\hline Delay duration (CS) & 9-bit & 0 to $511 \mu \mathrm{s}$ \\
\hline Repetition number (CS) & 12-bit & 0 to 4,095 times \\
\hline
\end{tabular}



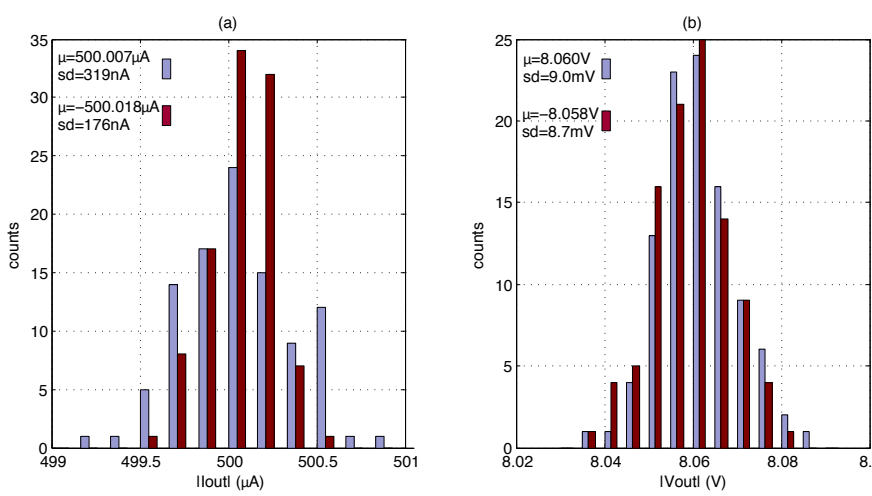

Fig. 7. Output stage mismatch analysis of the current conveyor (a) and the transimpedance (b)

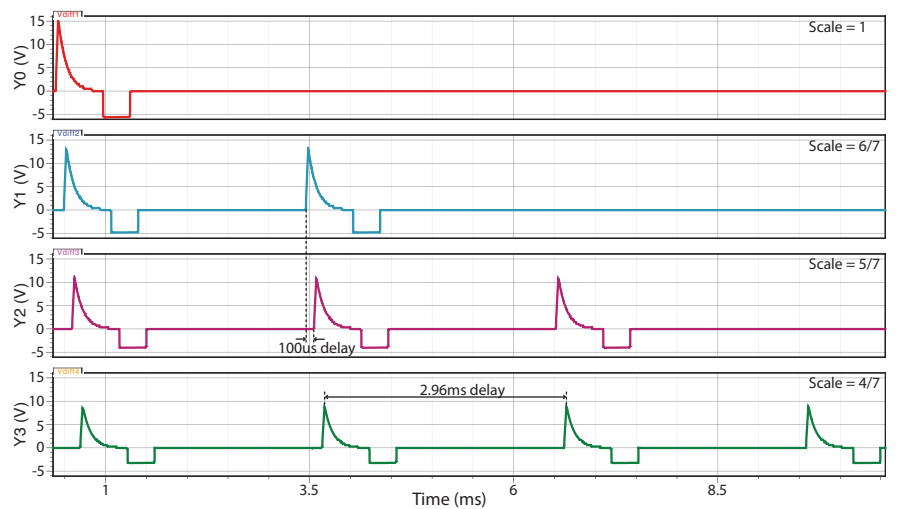

Fig. 8. 8-channel simulation results using a triangular exponential pulse

HighZ where the electrode is not driven and remains floating.

\section{Simulation Results}

The system has been simulated using AMS $0.18 \mu \mathrm{m}$ high voltage process. Monte-Carlo analysis have been run to characterize the matching between channels. Histograms in Fig. 7 show the mismatch distribution for both the current conveyor and the transimpedance when driving the maximum output for both signs. Matching of the different channels for both amplitude and sign is of particular interest as it is directly linked to the correct charge balancing of the stimulation and thus its safety. Here the system presents output standard deviations (sd) lower than $25 \%$ of the LSB and the difference between signs is lower than $1 \%$ for average values $(\mu)$.

In order to test the design, parameters have been sent via the SPI interface to the system to configure 8 channels. Channels are used in pairs; each pair of channels being connected to a $30 \mathrm{k} \Omega$ impedance. For this example, a triangular exponential decaying waveform has been loaded in the system. Simulation results are shown in Fig. 8. They demonstrate the ability of the system to drive simultaneously several channels with different scales, delays and number of repetitions between them. The voltage swing of the output stages is also shown, as the differential voltage between two channels has been pushed to $15 \mathrm{~V}$ for the largest scale of this simulation.

\section{CONCLUSiON}

In this work we have shown an 8-channel system which can interface to electrodes arrays. Both the analogue design and the digital circuit have been covered. Increasing the number of channels has been made particularly simple due to the unique bus between the main controller and the channels. Moreover, large matching has been achieved allowing accurate distribution of currents between channels.

Specific settings are available for each channel allowing deeper control of stimulations by clinicians. In particular the selection of different scales and delays between the channels allows experimenting electrical field shaping inducing more acute response of the tissue. The choice of the pulse waveform is a parameter which can be used to provide safer and more efficient stimulations strategy.

The next iteration of this work will involve fabricating this device then testing it's field shaping capabilities compared to modelling theory.

\section{REFERENCES}

[1] G. C. Albert, C. M. Cook, F. S. Prato, and A. W. Thomas, "Deep brain stimulation, vagal nerve stimulation and transcranial stimulation: An overview of stimulation parameters and neurotransmitter release," Neuroscience and Biobehavioral Reviews, vol. 33, no. 7, pp. 1042-1060, 72009.

[2] V. Valente, A. Demosthenous, and R. Bayford, "Electric field focusing and shifting technique in deep brain stimulation using a dynamic tripolar current source," in Circuits and Systems (ISCAS), Proceedings of 2010 IEEE International Symposium on, 2010, pp. 2091-2094.

[3] M. Bugbee, N. Donaldson, A. Lickel, N. Rijkhoff, and J. Taylor, "An implant for chronic selective stimulation of nerves." Proceedings of IFESS, 2008.

[4] V. M. Woods, I. F. Triantis, C. Agathos, and C. Toumazou, "Capacitive pulse shapes for platinum cuff electrodes," in 33rd Annual International IEEE EMBS Conference, 2011, pp. 5408-5411.

[5] K. W. Horch and G. Dhillon, Neuroprosthetics: Theory and Practice. World Scientific, 2004

[6] M. Ortmanns, A. Rocke, M. Gehrke, and H. Tiedtke, "A 232-channel epiretinal stimulator asic," IEEE Journal of Solid-State Circuits, vol. 47, no. $12,2007$.

[7] T. G. Constandinou, J. Georgiou, and C. Toumazou, "A partial-currentsteering biphasic stimulation driver for vestibular prostheses," IEEE Trans Biomed Circ Sys, vol. 2, no. 2, pp. 106-113, 2008.

[8] B. K. Thurgood, D. J. Warren, N. M. Ledbetter, G. A. Clark, and R. R. Harrison, "A wireless integrated circuit for 100-channel chargebalanced neural stimulation," Biomedical Circuits and Systems, IEEE Transactions on, vol. 3, no. 6, pp. 405-414, 2009.

[9] A. Eftekhar, T. Constandinou, D. Abbruzzese, V. Woods, I. Triantis, E. Drakakis, and C. Toumazou, "A programmable neural interface for investigating arbitrary stimulation strategies," IFESS, 2009.

[10] N. Laotaveerungrueng, R. R. Lahiji, S. L. Garverick, and M. Mehregany, "A high-voltage, high-current cmos pulse generator asic for deep brain stimulation," in Engineering in Medicine and Biology Society (EMBC), 2010 Annual International Conference of the IEEE, 2010, pp. 15191522.

[11] D. A. Wagenaar and S. M. Potter, "A versatile all-channel stimulator for electrode arrays, with real-time control," Journal of Neural Engineering, vol. 1 , no. 1 , p. $39,2004$.

[12] G. Loeb and R. Peck, "Cuff electrodes for chronic stimulation and recording of peripheral nerve activity," Journal of Neuroscience Methods, vol. 64, pp. 95-103, 1996.

[13] K. Bult and G. J. G. M. Geelen, "An inherently linear and compact mostonly current division technique," Solid-State Circuits, IEEE Journal of, vol. 27, no. 12, pp. 1730-1735, 1992.

[14] B. Linares-Barranco, T. Serrano-Gotarredona, and R. SerranoGotarredona, "Compact low-power calibration mini-dacs for neural arrays with programmable weights," Neural Networks, IEEE Transactions on, vol. 14, no. 5, pp. 1207-1216, 2003. 